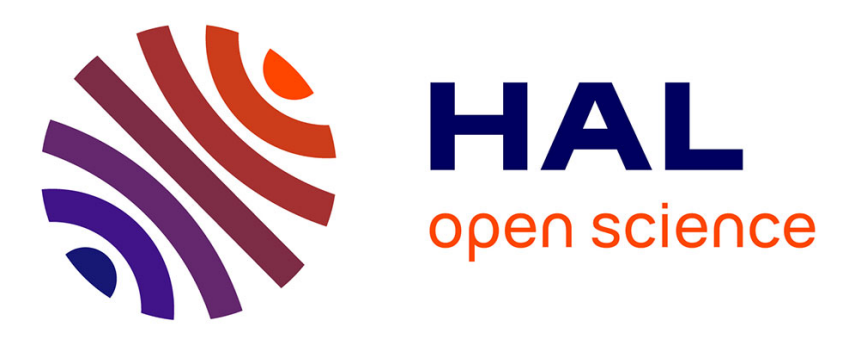

\title{
Dissipation and Thermoelastic Coupling Associated with Fatigue of Materials
}

\author{
André Chrysochoos, Thomas Boulanger, Anna Eva Morabito
}

\section{To cite this version:}

André Chrysochoos, Thomas Boulanger, Anna Eva Morabito. Dissipation and Thermoelastic Coupling Associated with Fatigue of Materials. Michel Frémond; Franco Maceri. Mechanics, Models and Methods in Civil Engineering, 61, Springer, pp.147-156, 2012, Lecture Notes in Applied and Computational Mechanics, 978-3-642-24637-1. 10.1007/978-3-642-24638-8_7 . hal-03338477

\section{HAL Id: hal-03338477 \\ https://hal.science/hal-03338477}

Submitted on 8 Sep 2021

HAL is a multi-disciplinary open access archive for the deposit and dissemination of scientific research documents, whether they are published or not. The documents may come from teaching and research institutions in France or abroad, or from public or private research centers.
L'archive ouverte pluridisciplinaire HAL, est destinée au dépôt et à la diffusion de documents scientifiques de niveau recherche, publiés ou non, émanant des établissements d'enseignement et de recherche français ou étrangers, des laboratoires publics ou privés. 


\title{
Dissipation and thermo-elastic coupling associated with fatigue of materials
}

\author{
André Chrysochoos ${ }^{1}$, Thomas Boulanger ${ }^{1}$, Anna Eva Morabito ${ }^{2}$ \\ 1 Laboratoire de Mécanique et Génie Civil, \\ Université Montpellier II, cc 048, \\ Place Eugène Bataillon, \\ 34095 Montpellier Cedex 5, France \\ 2 Dipartimento di Ingegneria dell'Innovazione, \\ Università à Degli Studi Di Lecce, \\ Viale Gallipoli, 49, \\ 73100 Lecce, Italia
}

\begin{abstract}
The fatigue behavior is examined in terms of calorimetric effects. Aluminum alloy and steel have been chosen as reference materials. Heat sources accompanying the fatigue mechanisms are derived from thermal images provided by an infrared camera. A processing method allows identifying separately thermoelastic and dissipative sources. Thermo-elastic effects are compared to theoretical predictions given by the basic, linear, isotropic thermo-elastic model. Dissipation amplitudes are analyzed as a function of the loading frequency and stress amplitude applied to the fatigue specimen. Finally, the heterogeneous character of the fatigue development is studied both in terms of thermo-elastic and dissipation sources.
\end{abstract}

\section{Introduction}

Fatigue design is based on the conventional fatigue limit classically obtained by using statistical processing of numerous tests which are particularly time consuming and expensive for industrial applications.

To get a rapid estimate of the fatigue limit, several research teams have monitored increases in the mean temperature of fatigue specimens [1-4]. The slope change of the warming regime occurring within certain level of loading range was empirically related to the fatigue limit corresponding with the chosen loading mode. Although realistic estimates of this limit are often obtained, the method still generates questionable results. Besides, it is basically problematic to consider the specimen heating as fatigue indicator: indeed, the temperature variations depend on the intensity and distribution of heat sources and also on heat diffusion within the material, and consequently on heat exchanges with the surroundings. This is the reason why the fatigue mechanisms is analyzed, in this paper, in terms of heat. The first work step is therefore to estimate the different heat sources by using thermal 
data provided by an infrared camera. A specific data processing has then been developed to estimate separately the dissipated energy coming from the irreversible evolutions of the micro-structural defects and the thermo-elastic coupling sources induced by the reversible thermal expansion of the crystalline network [5].

This paper is composed as follows: Section 2 is devoted to a brief recall of the thermo-mechanical framework used to introduce the different heat sources. Section 3 discusses different possible diffusion models and introduces the corresponding heat equation we used for the source computation. Section 4 describes the experimental arrangement, the infrared device and the data processing while Section 5 gives some experimental results.

\section{Calorimetric balance}

The framework of Generalized Standard Materials is used [6,7]. The equilibrium state of each volume material element can then be characterized by a set of $n$ state variables. The chosen state variables are $T$, the absolute temperature, $\varepsilon$, the SPH strain tensor, and $\left(\alpha_{1}, \ldots, \alpha_{n-2}\right)$, the $n-2$ scalar components of the vector $\alpha$ of "internal" variables that sum up the micro-structural state of the material. By construction, the thermodynamic potential is the Helmholtz free energy $\psi$. Combining both first and second principles of thermodynamics provides the local heat equation:

$$
\rho C \dot{T}-\operatorname{div}(k \cdot \operatorname{grad} T)=\left(\sigma-\rho \psi_{, \varepsilon}\right): \dot{\varepsilon}-\rho \psi_{, \alpha} \cdot \dot{\alpha}+\rho T \psi_{, T \varepsilon}: \dot{\varepsilon}+\rho T \psi_{, T \alpha} \cdot \dot{\alpha}+r_{e}
$$

The left-hand member consists of a differential operator applied to the temperature, while the right-hand member groups the different heat sources [8]. Here $\rho$ represents the mass density, $C$ the specific heat, $k$ the conduction tensor and $\sigma$ the Cauchy stress tensor. The different heat sources are in turn: the intrinsic dissipation $d_{1}=$ $\left(\sigma-\rho \psi_{, \varepsilon}\right): \dot{\varepsilon}-\rho \psi_{, \alpha} \cdot \dot{\alpha}$, the thermo-elastic source $s_{\text {the }}=\rho \psi_{, T \varepsilon}: \dot{\varepsilon}$, the "internal" coupling source $s_{\mathrm{ic}}=\rho \psi_{, T \alpha}: \dot{\alpha}$, and the external heat supply $r_{e}$. The following hypotheses were put forward for the infrared image processing to obtain the heat source estimate:

- Mass density and specific heat are material constants, independent of the thermodynamic state,

- Convective terms associated with the particular time derivative of the temperature are neglected because temperature gradients and displacement velocity amplitudes are low,

- The heat conduction tensor remains constant and isotropic during the test $\left(k_{i j}=k \delta_{i j}\right)$,

- The external heat supply $r_{e}$ due to heat exchange by radiation is time-independent, so the equilibrium temperature field $T_{0}$ verifies:

$-k \Delta T_{0}=r_{e}$, 
- Temperature variations induced by the fatigue test have no influence on the micro-structural state. Naturally this assertion becomes unsound as soon as the temperature variation rises to a high level. Small temperature variations will only be considered in what follows. In such a context, the coupling source $s_{\text {ic }}$ is neglected in equation (1) and the fatigue is considered as a purely dissipative mechanism accompanied by a classical thermo-elasticity.

Under the above hypotheses, the heat conduction equation could be rewritten in the following compact form:

$$
\rho C \frac{\partial \theta}{\partial t}-k \Delta \theta=d_{1}+s_{\text {the }}=s
$$

where $\theta=T-T_{0}$ symbolizes the temperature variation, while $s$ stands for the overall heat source.

\section{Towards simplified heat diffusion problems}

Determination of heat source fields within the whole gauge-part of the specimen should be an ultimate aim of the image processing. Unfortunately, for 3D cases, this operation is an ill-posed inverse problem that is impossible to solve without information on the source distribution to be quantified [9]. However, for thin, flat specimens, several options may be proposed. Let us note $L, l$ and $e$ as the length, width and thickness, respectively, of the specimen gauge part. The associated coordinates are in turn $x, y, z$.

The first option considers that the source averaged over the sample thickness is representative of what happens throughout the thickness. Integration of the heat equation according to this dimension then leads to a $2 \mathrm{D}$ diffusion problem. By noting $\bar{f}(x, y, t)=\frac{1}{e} \int_{-e / 2}^{+e / 2} f(x, y, z, t) \mathrm{d} z$ as the mean operation applied to $f$, we finally get:

$$
\rho C\left(\frac{\partial \bar{\theta}}{\partial t}+\frac{\bar{\theta}}{\tau_{\mathrm{th}}^{2 \mathrm{D}}}\right)-k\left(\frac{\partial^{2} \bar{\theta}}{\partial x^{2}}+\frac{\partial^{2} \bar{\theta}}{\partial y^{2}}\right)=\bar{s},
$$

where the approximation $\rho C \frac{\bar{\theta}}{\tau_{\mathrm{th}}^{2 \mathrm{D}}} \approx \frac{-k}{e}\left[\frac{\partial \theta}{\partial z}\right]_{-e / 2}^{+e / 2}$ claims linear uniform Fourier conditions at the specimen boundary. The time constant $\tau_{\mathrm{th}}^{2 \mathrm{D}}$ then characterizes heat losses through the specimen surfaces $z= \pm \frac{e}{2}$.

To improve the signal-to-noise ratio and reduce the number of numerical space derivations, we may assume that the mean heat sources associated with each crosssection $S$ of the specimen is relevant for describing what happens throughout $S$. Integrating the heat conduction equation over the cross-section $S$ then leads to a 1D thermal diffusion problem. 
Indeed, by noting $\overline{\bar{f}}(x, t)=\frac{1}{S} \int_{-l / 2}^{+l / 2} \int_{-e / 2}^{+e / 2} f(x, y, z, t) \mathrm{d} y \mathrm{~d} z$, the heat equation becomes:

$$
\rho C\left(\frac{\partial \overline{\bar{\theta}}}{\partial t}+\frac{\overline{\bar{\theta}}}{\tau_{\mathrm{th}}^{1 \mathrm{D}}}\right)-k\left(\frac{\partial^{2} \overline{\bar{\theta}}}{\partial x^{2}}\right)=\overline{\bar{s}}
$$

The approximation $-k\left(\overline{\overline{\frac{\partial^{2} \theta}{\partial y^{2}}+\frac{\partial^{2} \theta}{\partial z^{2}}}}\right) \approx \rho C \frac{\overline{\bar{\theta}}}{\tau_{\mathrm{th}}^{1 \mathrm{D}}}$ is once more based on the assumption that heat losses at the cross-section boundary $\left(y= \pm \frac{l}{2}, z= \pm \frac{e}{2}\right)$ are linear with respect to the temperature variation. To estimate the left-hand member of equation (5), thermo-profiles have to be built by averaging the temperature field over the specimen width. To make the method operational, these profiles are assumed to be close to the lengthwise temperature distribution $\overline{\bar{\theta}}(x, t)$.

A third option can be formulated to further simplify equation (1). Like stress and strain fields, we may suppose that, before localization onset, the heat source distribution is uniform at any time within the specimen gauge part. This is consistent with a classical view of homogeneous uniaxial tests. In such cases, the spectral solution of the heat equation can be analytically determined using eigen-functions of the Laplacian operator. For symmetric linear boundary conditions and initial conditions corresponding to uniform temperature fields, the spectral solution may then be well approximated by only considering the first eigen-function [8]. The heat equation becomes an ordinary differential equation and can be written as:

$$
\rho C\left(\frac{\mathrm{d} \theta}{\mathrm{d} t}+\frac{\theta}{\tau_{\mathrm{eq}}}\right) \approx s
$$

where $\theta$ is now the temperature variation measured at the center of the specimen gauge part, with $\tau_{\text {eq }}$ characterizing all local heat losses. Practically $\theta$ is identified to the mean temperature of a small centered area (e.g., $\left.2 \times 2 \mathrm{~mm}^{2}\right)$.

\section{Experimental procedure}

The fatigue machine used in the present work is a servo-hydraulic machine with a load cell of $\pm 25 \mathrm{kN}$. Tests were conducted on thin, flat specimens which have been designed to optimize the loading capacity of the testing machine at a maximum loading frequency $\left(\max \left(f_{\mathrm{L}}\right)=50 \mathrm{~Hz}\right)$. The size of the gauge part of these specimens is $2.5 \times 10 \times 10 \mathrm{~mm}^{3}$.

Thermography detection was performed using a CCD infrared camera (CEDIP $\mathrm{SW}$ ). During the tests, the lens axis of the camera was kept fixed and perpendicular to the surface of the specimen. The maximum frame-rate of the IR camera reached 250 images per second (i.e., maximum sampling frequency $\max \left(f_{\mathrm{S}}\right)=250 \mathrm{~Hz}$ ) for an image resolution of $64 \times 128$ pixels. This corresponds to a space resolution of about $0.2 \mathrm{~mm} /$ pixel which enables us to observe the whole gauge-part of the specimen. 


\subsection{Testing}

Within the above-mentioned limits imposed by the specimen geometry and machine capacity, it was possible to carry out load-control fatigue tests near the fatigue limit for two load ratios, $R_{\sigma}=0$ and $R_{\sigma}=0.1$, with the loading path being a pure sine until $50 \mathrm{~Hz}$. The fatigue tests were set up in blocks of a limited number of cycles, compatible with the finite RAM capacities of the IR device as regards the maximum number of recorded images and time necessary to download these infrared images. Each block consisted of 20,000 cycles performed at constant loading frequency $f_{\mathrm{L}}$, constant load ratio $R_{\sigma}$, and constant stress range $\Delta \sigma$. At the end of each block, the recorder of the infrared camera was switched off and the loading stopped. Between two blocks, we waited about $10 \mathrm{~min}$ in order to restart at the thermal equilibrium. For a single specimen, the range $\Delta \sigma$ may vary between blocks.

\subsection{Image processing}

The linearity of equations (4-6) and that of boundary conditions enabled us to separately analyze the influence of both sources on the temperature pattern. We then defined $\theta_{\text {the }}$ and $\theta_{\mathrm{d}}$ induced by $s_{\text {the }}$ and $d_{1}$, respectively.

The thermo-elastic behavior is assumed to be linear and isotropic. By noting $\lambda$ the linear thermal expansion coefficient, $K$ the elastic bulk modulus, we can verify, for the chosen steel and Duralumin, that the ratio:

$$
\frac{3 K \lambda^{2}}{\rho C} T_{0} \ll 1
$$

In such a case, for cyclic loading of sinusoidal form

$$
\sigma(t)=\sigma_{\mathrm{m}}+\frac{\Delta \sigma}{2} \sin \left(2 \pi f_{\mathrm{L}} t\right)
$$

the following properties of the thermo-elastic source can be derived:

- $s_{\text {the }}$ is a periodic signal whose frequency spectrum is reduced to $f_{\mathrm{L}}$.

- the thermo-elastic energy vanishes at the end of each complete loading cycle:

$$
w_{\text {the }}=\oint_{\text {Cycle }} s_{\text {the }} \mathrm{d} \tau=0
$$

In what follows, we will note respectively $\Delta s_{\text {the }}$ and $\Delta \theta_{\text {the }}$ the mean ranges of $s_{\text {the }}$ and $\theta_{\text {the }}$ associated with a given number $n$ of complete cycles.

Concerning the dissipation, the temperature variation $\theta_{\mathrm{d}}$ induced by $d_{1}$ must be positive according to Eqs (4-6). Besides, equation (1) also shows that the dissipated 
energy may come from both the strain-rate $\dot{\varepsilon}$ and $\dot{\alpha}$, which stands for the microstructural evolution. Assuming that the degradation of the microstructure during small number $n$ of cycles is low, we will consider the mean dissipation to analyze the fatigue kinetics:

$$
\bar{d}_{1}=\oint_{n \text { Cycles }} n^{-1} f_{\mathrm{L}} d_{1} \mathrm{~d} \tau
$$

To estimate the heat sources, a local least-squares fitting of the thermal data has been chosen. The local approximation function has the following form:

$$
\theta^{\mathrm{app}}(x, t)=\underbrace{p_{1}(x) t+p_{2}(x)}_{\text {drift }}+\underbrace{p_{3}(x) \cos \left(2 \pi f_{\mathrm{L}} t\right)+p_{4}(x) \sin \left(2 \pi f_{\mathrm{L}} t\right)}_{\text {periodic response }} .
$$

The trigonometric time functions describe the periodic part of the thermoelastic effects while the linear part takes into account transient effects due to heat losses, dissipative heating and possible drifts of the equilibrium temperature. The functions $p_{i}(x), i=1, \ldots, 4$, are:

- second order polynomials of $x$ and $y$ (2D analyze, Eq. (4)),

- second order polynomials of $x$ (1D analyze, Eq. (5)),

- constants (local analyze, Eq. (6)).

For instance, the one-dimensional data processing introduces $3 \times 4=12$ parameters $P_{1}, \ldots, P_{12}$ for each space-time fitting window. The corresponding expressions of $\Delta s_{\text {the }}$ and $\bar{d}_{1}$ are respectively:

$$
\begin{aligned}
& \Delta s_{\text {the }}=2 \sqrt{\begin{array}{l}
{\left[\frac{I^{2}}{N_{\mathrm{p}}}\left(2 \pi f_{\mathrm{L}} P_{1}+\frac{P_{2}}{\tau_{\mathrm{th}}^{1 \mathrm{D}}}\right)+2 \pi f_{\mathrm{L}} P_{9}-\frac{2 k P_{2}}{\rho C \Delta x^{2}}+\frac{P_{10}}{\tau_{\mathrm{th}}^{1 \mathrm{D}}}\right]^{2}} \\
+\left[\frac{I^{2}}{N_{\mathrm{p}}}\left(\frac{P_{1}}{\tau_{\mathrm{th}}^{1 \mathrm{D}}}-2 \pi f_{\mathrm{L}} P_{2}\right)-2 \pi f_{\mathrm{L}} P_{10}-\frac{2 k P_{1}}{\rho C \Delta x^{2}}+\frac{P_{9}}{\tau_{\mathrm{th}}^{1 \mathrm{D}}}\right]^{2}
\end{array}} \\
& \bar{d}_{1}=\frac{I^{2}}{N_{\mathrm{p}}}\left(\frac{P_{3}}{\Delta t}+\frac{P_{4}}{\tau_{\mathrm{th}}^{1 \mathrm{D}}}\right)-2 \frac{k P_{4}}{\rho C \Delta x^{2}}+\frac{P_{11}}{\Delta t}+\frac{P_{12}}{\tau_{\mathrm{th}}^{1 \mathrm{D}}}
\end{aligned}
$$

where $N_{\mathrm{p}}=2 \times N_{0}+1$ is the number of pixels, $I^{2}=\sum_{i=-N_{0}}^{i=N_{0}} i^{2}, \Delta x$ is the space resolution of the camera, $\Delta t$ the time-step associated with the frame-rate (i.e., sampling frequency $f_{\mathrm{S}}$ ). 


\section{Experimental results}

In this part, the results concern the load ratios $R_{\sigma}=0$ for steel and $R_{\sigma}=0.1$ for Duralumin. Note, however, that similar findings were obtained for a load ratio $R_{\sigma}=-1$. We also stress that the following results are derived using the $1 \mathrm{D}$ analysis of longitudinal thermoprofiles (Eqs (12-13)) and the 2D analysis to construct spatial distribution of $\Delta s_{\text {the }}$. As previously mentioned, each stress range $\Delta \sigma$ is associated with a loading block of 20,000 cycles. Apart from the last thousands of cycles corresponding to the fatigue crack growth, we observed distributions of heat sources which slowly develop. Mean values were then computed by averaging the source over each longitudinal profile constructed during a block. When divided by $\rho C$, the source unit becomes ${ }^{\circ} \mathrm{C} . \mathrm{s}^{-1}$. This gives an equivalent warming speed corresponding to a monotonous thermal process in adiabatic conditions, and facilitates the comparison of both materials.

\subsection{Thermo-elastic sources}

In figure 1, the mean thermoelastic source within the gauge part of the specimen was plotted as a function of the stress range. First note the order of magnitude of this coupling source for a loading performed at $f_{\mathrm{L}}=50 \mathrm{~Hz}$ : we get about $100{ }^{\circ} \mathrm{C} . \mathrm{s}^{-1}$ for both materials.

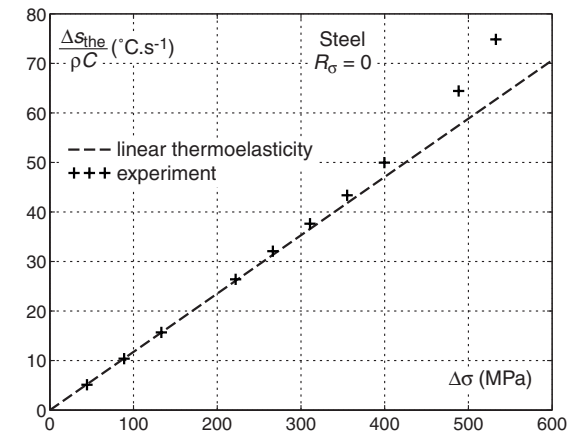

a

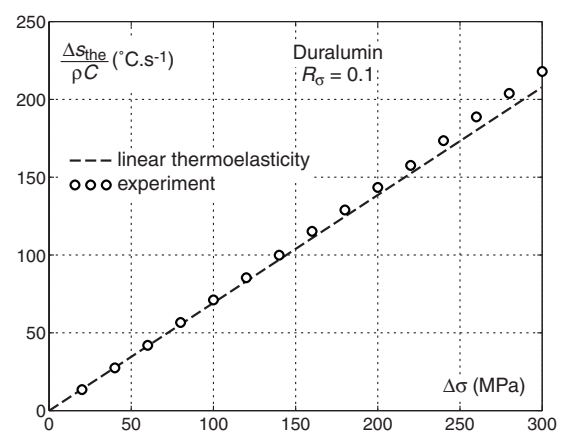

$\mathrm{b}$

Fig. 1. Thermoelastic source versus stress range at $f_{\mathrm{L}}=50 \mathrm{~Hz}$. a) steel: $\left.\sigma_{\mathrm{e}}=360 \mathrm{MPa}, \mathrm{b}\right)$ Duralumin: $\sigma_{\mathrm{e}}=310 \mathrm{MPa}$.

The experimental data can also be compared with theoretical predictions derived from the basic linear, isotropic thermoelastic model. We observed pretty good predictions for small stress ranges while, close to the yield stress $\sigma_{\mathrm{e}}$, both sets of data gradually diverge. The gradual drift of experimental data may be interpreted in terms of non linear thermoelasticity as long as the mechanical test remains homogeneous. In such a case however, strains, stresses, and heat sources must be uniformly distributed within the gauge part of the sample. This condition will be examined in section 5.3. 


\subsection{Dissipative sources}

In figure 2 the mean dissipation per block was also plotted as a function of the stress range. The dissipated energy-rates are between 100 and 1000 times lower than those associated with the thermoelastic couplings.
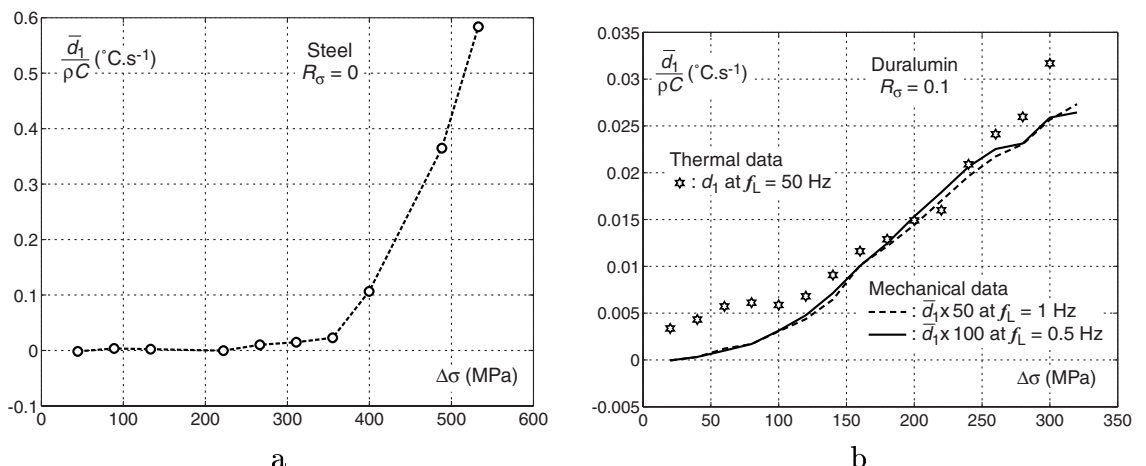

Fig. 2. Intrinsinc dissipation versus stress range. a) steel, b) Duralumin.

The dissipation is particularly weak in the case of aluminum alloy (around $10^{-2}{ }^{\circ} \mathrm{C} . \mathrm{s}^{-1}$ at $f_{\mathrm{L}}=50 \mathrm{~Hz}$ ). In practice, we were unable to get plausible dissipation during tests performed at loading frequencies less than $50 \mathrm{~Hz}$ because of bad signalto-noise ratios. In order to check the consistency of the image processing, mechanical estimates of the dissipation were performed on aluminum samples. Therefore, we identified the intrinsic dissipation with the hysteresis area of the mechanical stressstrain loops. Fatigue tests were made at low loading frequencies $\left(f_{\mathrm{L}}=0.5-1 \mathrm{~Hz}\right)$ to avoid sliding of the extensometer clips. The comparison between thermal and mechanical estimates was made by assuming the dissipation proportional to $f_{\mathrm{L}}$. Results shown in figure $2 \mathrm{~b}$ are in quite good agreement with such a hypothesis.

\subsection{Heterogeneous distribution of heat sources}

The figure 3 respectively shows the 1D distribution of $\Delta s_{\text {the }}$ and $\bar{d}_{1}$ for 10 blocks performed on a steel sample, increasing the stress range from one block to another. In figure 3a we observe that the non linearity of $\Delta s_{\text {the }}$ vs $\Delta \sigma$ is accompanied by a non uniform distribution of sources. The dissipation profiles are shown in figure $3 \mathrm{~b}$. One detects zones (i.e., cross-sections) which dissipate energy more than others. These zones are fixed from one block to another and one of them will be the locus of the crack inception. In figure $4,2 \mathrm{D}$ distributions of $\Delta s_{\text {the }}$ are plotted. We chose 4 maps related to blocks 1, 7 and 15. They show a heterogeneous distribution of thermoelastic sources that takes place since the beginning of the test. Regarding the two maps associated with block 15, the first one is selected at the beginning of block 15, several thousands of cycles before the crack inception. The second one shows the influence of the crack propagation. 


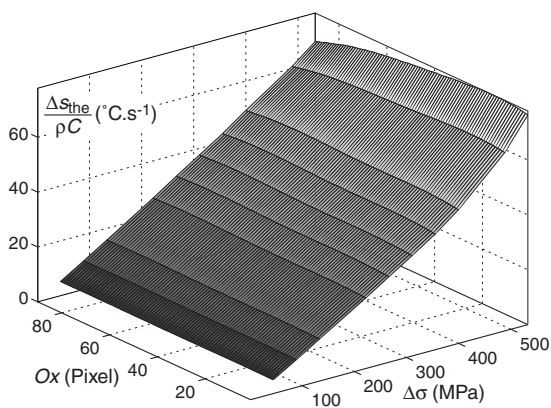

a

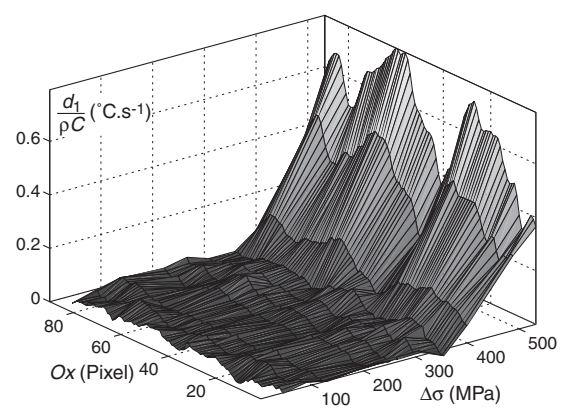

$\mathrm{b}$

Fig. 3. Spatial distribution of heat sources.

a) longitudinal profiles of thermoelastic sources as a function of stress range, b) longitudinal profiles of dissipation sources as a function of stress range.

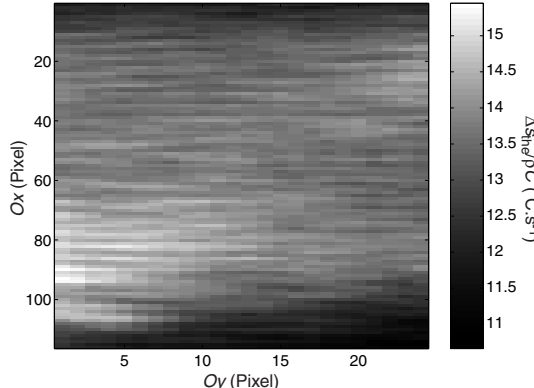

a

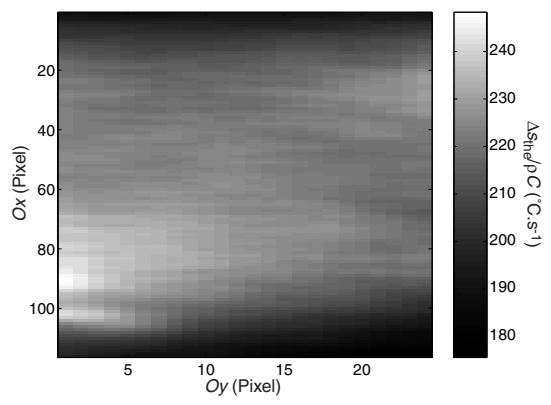

c

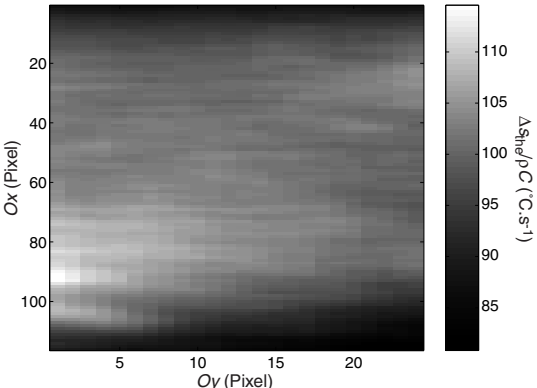

$\mathrm{b}$

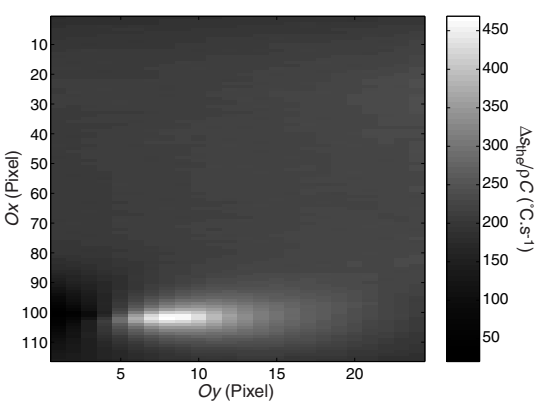

d

Fig. 4. 2D distributions of thermoelastic sources.

a) block 1, b) block 7, c) block 15, d) block 15: influence of crack growth. $O x$ : length, $O y$ : width.

\section{Concluding comments}

In this study, the fatigue of two classical metals is examined in terms of thermal and calorimetric effects. The image processing enables us to compute separately thermoelastic sources and dissipation. We detect very low dissipation intensities 
compared to the amplitudes of thermoelastic sources, particularly for Duralumin where the ratio $\bar{d}_{1} / \Delta s_{\text {the }}$ reaches down to $10^{-3}$. For low stress ranges, we observe a good agreement between thermoelastic sources derived from experimental data and those predicted by the linear, isotropic thermoelastic model. Near the yield stress, the divergence of results is questionable. This may come from a non linear material behavior effect, but also from a non linear structure effect. Indeed, the main result we would like to underline is the heterogeneous character of the fatigue test which is highlighted here in terms of heat sources distribution. Check tests have to be performed in a near future using digital image correlation combined with infrared techniques. The aim will be to verify if zones where sources intensity is the more important are correlated with zones where strain localization develops.

\section{References}

1. Luong M.P. (1998) Fatigue limit evaluation of metals using an infrared thermographic technique. Mech. Mater., 28, 155-163.

2. La Rosa G. and Risitano A. (2000) Thermographic methodology for rapid determination of the fatigue limit of materials and mechanical components. Int. J. Fatigue, 22, 65-73.

3. Yang B., Liaw P.K., Wang H., Jiang L., Huang J.Y., Kuo R.C. and Huang J.G. (2001) Thermographic investigation of the fatigue behavior of reactor pressure vessel steels. Mater. Sci. Eng. A, 314, 131-139.

4. Krapez J.C., Pacou D. and Gardette G. (2000) Lock-in thermography and fatigue limit of metals. In: Balageas D.,Beaudoin J.L., Busse G. and Carlomagno G.M. (Eds.) Quantitative Infrared Thermography 5: QIRT', 277-282.

5. Boulanger T., Chrysochoos A., Mabru C. and Galtier A. (2003) Analysis of heat sources induced by fatigue loading. In: Varvani-Farahani, A. and Brebbia, C.A. (Eds.) $1^{\text {st }}$ International Conference on Fatigue Damage of Materials: Experimental and Analysis, 255-262.

6. Germain P., Nguyen Q.S. and Suquet P. (1983) Continuum thermodynamics. J. Appl. Mech., 50, 1010-1020.

7. Lemaitre J. and Chaboche J.L. (1990) Mechanics of solid materials, Cambridge University Press.

8. Chrysochoos A. and Louche H. (2000) An infrared image processing to analyse the calorific effects accompanying strain localisation. Int. J. Eng. Sci., 38, 17591788.

9. Capatina A. and Stavre R. (2000) Algorithms and convergence results for an inverse problem in heat propagation. Int. J. Eng. Sci., 38, 575-587. 